Homology, Homotopy and Applications, vol.22(2), 2020, pp.279-293

\title{
SO, WHAT IS A DERIVED FUNCTOR?
}

\author{
VLADIMIR HINICH
}

(communicated by Emily Riehl)

\begin{abstract}
We rethink the notion of derived functor in terms of correspondences, that is, functors $\mathcal{E} \rightarrow[1]$. While derived functors in our sense, when they exist, are given by Kan extensions, their existence is a strictly stronger property than the existence of Kan extensions. We show, however, that derived functors exist in the cases one expects them to exist. Our definition is especially convenient for the description of a passage from an adjoint pair $(F, G)$ of functors to a derived adjoint pair $(\mathbf{L} F, \mathbf{R} G)$. In particular, canonicity of such a passage is immediate in our approach. Our approach makes perfect sense in the context of $\infty$-categories.
\end{abstract}

\section{Introduction}

This is a new rap on the oldest of stories Functors on abelian categories.

If the functor is left exact

You can derive it and that's a fact.

But first you must have enough injective

Objects in the category to stay active.

If that's the case no time to lose;

Resolve injectively any way you choose.

Apply the functor and don't be sore -

The sequence ain't exact no more.

Here comes the part that is the most fun, Sir,

Take homology to get the answer.

On resolution it don't depend:

All are chain homotopy equivalent.

Hey, Mama, when your algebra shows a gap

Go over this Derived Functor Rap.

P. Bressler, Derived Functor Rap, 1988

Received January 20, 2019, revised July 26, 2019, December 3, 2019; published on May 6, 2020. 2010 Mathematics Subject Classification: 18G10, 18 G55.

Key words and phrases: derived functor, $\infty$-category.

Article available at http://dx.doi.org/10.4310/HHA.2020.v22.n2.a18

Copyright (C) 2020, Vladimir Hinich. Permission to copy for private use granted. 


\subsection{Advertisement}

Our approach to derived functors can be explained in one sentence.

In the language of cocartesian fibrations over [1], calculation of a left derived functor becomes a localization.

This sentence is, actually, a recipe:

- Convert a functor $f: \mathcal{C} \rightarrow \mathcal{D}$ into a cocartesian fibration $p: \mathcal{E} \rightarrow[1]$.

- Localize $\mathcal{E}$.

- If the localization $\mathcal{E}^{\prime}$ of $\mathcal{E}$ remains a cocartesian fibration over [1], we say that $f$ has a left derived functor; this is the functor classifying $\mathcal{E}^{\prime}$.

One similarly treats the right derived functors as well as the derived functors of an adjoint pair of functors.

In this paper we argue that this approach leads to a very good notion of derived functor. We show that, given an adjoint pair of functors, their respective derived functors, if they exist, are automatically adjoint. We also show that the derived functors defined in this way behave nicely in families, as explained in Subsection 1.4.1 below.

\subsection{A bit of history}

The prehistoric understanding of derived functors, based on the existence of resolutions, is beautifully described in the epigraph. While this description makes perfect sense, it cannot possibly serve as a definition; it is merely a construction.

A historic period starts with the reformulation of derived functors in terms of localization of categories, performed by Grothendieck and Verdier in the abelian setting, and by Quillen in topology. It was first documented in Hartshorne's notes [RD]. The idea of this approach is that, in order to make sense of arbitrary choices of resolutions, one has to construct a category where an object of an abelian category and its resolution become isomorphic; this is the derived category, and it is constructed by localizing the category of complexes. A similar idea led Quillen $[\mathbf{Q . H A}]$ to define model categories and their localizations, homotopy categories.

Using the language of localization, derived functors are defined by a universal property: according to Hartshorne [RD, 1.5], and Quillen [Q.HA, 4.1], a left derived functor can be defined as (what is nowadays called) the right Kan extension, whereas a right derived functor can be defined as the left Kan extension.

A similar approach is used for defining derived functors in the context of $\infty$-categories, see Cisinski $[\mathbf{C}]$.

Another definition of derived functor was suggested by Deligne in his report on étale cohomology with proper support, see $[\mathbf{D}]$, in the context of triangulated categories. The value of a left derived functor, according to Deligne, is a pro-object of the respective localization. If the values of a derived functor so defined are corepresentable, then the derived functor is a right Kan extension of the original functor. However, the existence of a right Kan extension does not seem to imply corepresentability of Deligne's derived functor. 


\section{2 .}

The definition of derived functors via Kan extensions is not, in our opinion, fully satisfactory. Here is one of the problems. The functors one has to derive often come in pairs. ${ }^{1}$ And, given an adjoint pair of functors, one expects them to give rise to an adjoint pair of derived functors. Each separate derived functor has a universal description as a Kan extension; but an adjoint pair is not just a pair of functors: to define an adjunction one also needs to specify a unit or a counit of the adjunction. This cannot be deduced in general from the description of derived functors as Kan extensions.

\subsection{Summary}

In Section 2.3 we define, following the recipe explained in 1.0, left and right derived functors. Our definition immediately implies that, for $f$ left adjoint to $g$, if $\mathbf{L} f$ and $\mathbf{R} g$ exist, they are automatically adjoint.

In Section 3 we describe the category of correspondences and its full subcategory of cocartesian correspondences.

The main results are proven in Section 4.

They include:

Corollary 4.2 saying that the left derived functor, in the sense of Definition 2.1, if it exists, is a right Kan extension.

A sufficient condition for existence of left (right) derived functors, including the case of left (right) Quillen functors.

In Section 4.2 we study the properties of diagrams of derived functors. The details are explained in Subsection 1.4.1 below.

In Section 4.3 we show that Deligne's definition of derived functors, given in [D], is a special case of our definition.

In Section 4.4 we show that, if $f^{\prime}: \mathcal{C}^{\prime} \rightarrow \mathcal{D}^{\prime}$ is $\infty$-categorical derived functor, passage to the respective homotopy categories defines a derived functor with respect to conventional localizations.

\subsection{Remarks}

1. The derived functors defined by Deligne are known to automatically preserve adjunction, see Keller's [K, 13.6].

2. In 2007 George Maltsiniotis [Mal] made a beautiful observation: if Kan extensions $\mathbf{L} f$ and $\mathbf{R} g$ of an adjoint pair of functors $(f, g)$ are absolute, $\mathbf{L} f$ and $\mathbf{R} g$ acquire an automatic adjunction. The same holds for an adjunction of infinity categories, see $[\mathbf{C}]$. In particular, Quillen's adjunction leads to absolute Kan extensions, so the derived functors between the infinity categories underlying a Quillen pair, are automatically adjoint. Derived functors in the sense of Definition 2.1 are, actually, absolute.

We are not sure, however, that defining derived functors as absolute Kan extensions would yield a notion preserving diagrams of derived functors as in Subsection 1.4.1 below.

\footnotetext{
${ }^{1}$ adjoint pairs.
} 


\subsubsection{Diagrams of derived functors}

It is known that the derived functor of a composition is not necessarily a composition of derived functors; it turns out, however, that this is the only obstacle to functoriality of the passage to derived functors; in Section 4.2 we prove that, given a ( $\infty$-)functor $F: B \rightarrow$ Cat and an appropriate collection of $\left(\infty\right.$-)subcategories $\mathcal{W}_{b} \subset F(b), b \in B$, so that for any arrow $a: b \rightarrow b^{\prime}$ in $B$ the derived functor of $F(a)$ exists, and is compatible with compositions, then the family of derived functors $\mathbf{L} F(a)$ "glue" to a functor $\mathbf{L} F: B \rightarrow$ Cat carrying $b \in B$ to the localization $F(b)^{\prime}$ of $F(b)$ with respect to $\mathcal{W}_{b}$, and any arrow $a: b \rightarrow b^{\prime}$ to $\mathbf{L} F(a): F(b)^{\prime} \rightarrow F\left(b^{\prime}\right)^{\prime}$.

We expect this property will be useful in studying higher descent in style of [HS] and $[\mathbf{M e}]$.

\section{Acknowledgments}

This work, being formally independent of George Maltsiniotis' [Mal], stems from a similar dissatisfaction with the existing definition of derived functor. We are grateful to him for bringing our attention to this work. We are grateful to B. Keller who explained to us that Deligne's definition leads to automatic adjunction of the derived functors. We are also grateful to D.-C. Cisinski who informed us about his book [C]. Discussions with Ilya Zakharevich were very useful. We are very grateful to the referee for his request to clarify some sloppy passages in the original version of the paper. The present work was partially supported by ISF grants 446/15 and 786/19.

\section{Left and right derived functors}

We will now present our definition of derived functors and formulate the main results.

In what follows the word "category" means infinity category, and "conventional category" means a category in the conventional sense. Our way of dealing with infinity categories is model-independent in the following sense. We work in the (infinity) category of (infinity) categories Cat. All properties of categories or of morphisms in categories we use are invariant under equivalences. In particular, all limits and colimits in this paper are in the sense of infinity categories. Since Quillen equivalence of model categories gives rise to equivalent infinity categories, and all existing models for infinity categories are Quillen equivalent to each other, we can use existing results proven in specific models to claim properties of our "model-independent" Cat. See more details about this approach in [H.EY, Section 2].

\section{1.}

A functor $f: \mathcal{C} \rightarrow \mathcal{D}$ can be converted, via the Grothendieck construction, to a cocartesian fibration $p: \mathcal{E} \rightarrow[1]$.

One has $\mathcal{E}=(\mathcal{C} \times[1]) \sqcup^{\mathcal{C}} \mathcal{D}$, with the map $\mathcal{C} \rightarrow \mathcal{D}$ given by $f$, see $[$ H.lec, 9.8]. Here is a description of $\mathcal{E}$ for conventional categories:

- $\mathrm{Ob}(\mathcal{E})=\mathrm{Ob}(\mathcal{C}) \sqcup \mathrm{Ob}(\mathcal{D})$. 
- $\operatorname{Hom}_{\mathcal{E}}(x, y)= \begin{cases}\operatorname{Hom}_{\mathcal{C}}(x, y), & x, y \in \mathcal{C}, \\ \operatorname{Hom}_{\mathcal{D}}(x, y), & x, y \in \mathcal{D}, \\ \operatorname{Hom}_{\mathcal{D}}(f(x), y), & x \in \mathcal{C}, y \in \mathcal{D}, \\ \emptyset, & x \in \mathcal{D}, y \in \mathcal{C} .\end{cases}$

The cocartesian fibration classified by the functor $f: \mathcal{C} \rightarrow \mathcal{D}$ will be denoted $p_{f}: \mathcal{E}_{f} \rightarrow[1]$.

\section{2.}

Similarly, a functor $f: \mathcal{C} \rightarrow \mathcal{D}$ can be converted to a cartesian fibration $q_{f}: \mathcal{F}_{f} \rightarrow[1]$ given by the formula $\mathcal{F}_{f}=\mathcal{D} \sqcup^{\mathcal{C}}(\mathcal{C} \times[1])$. Its fibers at 0 and 1 are $\mathcal{D}$ and $\mathcal{C}$ respectively.

\subsection{Derived functors}

\subsubsection{Localization}

Recall that the localization of a category $\mathcal{C}$ with respect to a subcategory $\mathcal{W}$ (we assume $\mathcal{M}$ contains the maximal subspace $\mathcal{C}^{e q}$ of $\mathcal{C}$ ) is defined by a universal property: the functor $\mathcal{C} \rightarrow \mathcal{C}\left[\mathcal{W}^{-1}\right]$ is an initial object among functors carrying $\mathcal{W}$ to equivalences. Localization is functorial in the following sense. Define $\mathrm{Cat}^{+}$to be the category of marked categories, that is pairs $\left(\mathcal{C}, \mathcal{C}^{\circ}\right)$ where $\mathcal{C}^{\circ}$ is a subcategory of $\mathcal{C}$ containing $\mathcal{e}^{e q}$. Then localization defines a functor $\mathcal{L}: \mathrm{Cat}^{+} \rightarrow$ Cat left adjoint to the functor ${ }^{2}$ $\mathcal{(} \mapsto\left(\mathcal{C}, \mathcal{C}^{e q}\right)$.

\subsection{2.}

Let $f: \mathcal{C} \rightarrow \mathcal{D}$ be a functor. Let $q_{\mathcal{C}}: \mathcal{C} \rightarrow \mathcal{C}^{\prime}$ and $q_{\mathcal{D}}: \mathcal{D} \rightarrow \mathcal{D}^{\prime}$ be localizations ${ }^{3}$ defined by subcategories $W_{\mathcal{C}}$ and $W_{\mathcal{D}}$ of $\mathcal{C}$ and $\mathcal{D}$ respectively. Denote by $\mathcal{E}_{f}^{\prime}$ the localization of $\mathcal{E}_{f}$ with respect to $W_{\mathcal{C}} \cup W_{\mathcal{D}}$.

Definition 2.1. If $\mathcal{E}_{f}^{\prime}$ is a cocartesian fibration over [1], we define a left derived functor $\mathbf{L} f: \mathcal{C}^{\prime} \rightarrow \mathcal{D}^{\prime}$ as the functor classifying the cocartesian fibration $\mathcal{E}_{f}^{\prime}$.

Right derived functors are defined similarly. Denote by $\mathcal{F}_{f}^{\prime}$ the localization of the cartesian fibration $\mathcal{F}_{f}$ with respect to $W_{\mathcal{C}} \cup W_{\mathcal{D}}$.

Definition 2.2. If $\mathcal{F}_{f}^{\prime}$ is a cartesian fibration over [1], we define a right derived functor $\mathbf{R} f: \mathcal{C}^{\prime} \rightarrow \mathcal{D}^{\prime}$ as the functor classifying the cartesian fibration $\mathcal{F}_{f}^{\prime} \rightarrow[1]$.

We will discuss in Section 4 the existence of derived functors defined in Definitions 2.1 and 2.2 and compatibility of these notions with the known ones.

The application to deriving adjunction is immediate.

Proposition 2.3. Let $f: \mathcal{C} \rightleftarrows \mathcal{D}: g$ be an adjoint pair of functors. Let $q: \mathcal{C} \rightarrow \mathcal{C}^{\prime}$ and $q: \mathcal{D} \rightarrow \mathcal{D}^{\prime}$ be localizations. Assume that the derived functors $\mathbf{L} f$ and $\mathbf{R} g$ exist. Then they form an adjoint pair.

In fact, $\mathcal{E}_{f}=\mathcal{F}_{g}$ and so $\mathcal{E}_{f}^{\prime}=\mathcal{F}_{g}^{\prime}$.

\footnotetext{
${ }^{2}$ This is the most general notion of localization in infinity categories. We called it Dwyer-Kan localization, see $[\mathbf{H . L}]$, in order to stress the origin of this notion.

${ }^{3}$ Without loss of generality we can think that $\mathcal{D}=\mathcal{D}^{\prime}$.
} 


\section{Correspondences and Kan extension}

The key to understanding derived functors lies in the category of correspondences Cor and its full subcategory Cor $^{c o c}$ of cocartesian correspondences.

\subsection{Correspondences}

Recall that a correspondence from $\mathcal{C}$ to $\mathcal{D}$ is given by a functor $\mathcal{C} \times \mathcal{D}^{\mathrm{op}} \rightarrow \mathcal{S}$, where $\mathcal{S}$ is the category of spaces. Equivalently, a correspondence can be defined as a functor $\mathcal{D} \rightarrow P(\mathcal{C})$ to presheaves of $\mathcal{C}$, or, vice versa, as a functor $\mathcal{C}^{o p} \rightarrow P\left(\mathcal{D}^{\mathrm{op}}\right)$.

Equivalently, a correspondence from $\mathcal{C}$ to $\mathcal{D}$ can be encoded into a functor $p: \mathcal{E} \rightarrow$ [1], together with the equivalences $\mathcal{C} \stackrel{\sim}{\rightarrow} \mathcal{E}_{0}, \mathcal{D} \stackrel{\sim}{\rightarrow} \mathcal{E}_{1}$, see [L.T, 2.3.1.3] and [H.L, 9.10]. A detailed proof of this fact (in a greater generality) can be found in $[\mathbf{H . E Y}$, Section 8].

We define Cor $=\mathrm{Cat}_{/[1]}$ the category of correspondences and Cor ${ }^{c o c}$ its full subcategory spanned by cocartesian fibrations over [1]. Note that arrows in $\operatorname{Cor}^{c o c}$ do not necessarily preserve cocartesian arrows.

\subsection{1.}

The map $\{0\} \sqcup\{1\} \rightarrow[1]$ defines, by the base change, a functor $\partial=\left(\partial_{0}, \partial_{1}\right):$ Cor $\rightarrow$ Cat $\times$ Cat. Correspondences can be composed: if $X, Y \in$ Cor and $\mathcal{D}:=\partial_{1}(X)=\partial_{0}(Y)$, the composition $Y \circ X$ is defined as follows. The category $Z:=X \sqcup^{\mathcal{D}} Y$ is endowed with a map to [2]; one defines $Y \circ X$ as the base change of $Z$ with respect to $\delta^{1}:[1] \rightarrow[2]$.

We denote by the same letter the restriction of $\partial$ to $\operatorname{Cor}^{c o c}$; the fiber at $(\mathcal{C}, \mathcal{D})$ is denoted by $\operatorname{Cor}_{\mathcal{C}, \mathcal{D}}^{c o c}$. The assignment $(\mathcal{C}, \mathcal{D}) \mapsto \operatorname{Cor}_{\mathcal{C}, \mathcal{D}}^{c o c}$ is covariant in $\mathcal{D}$ and contravariant in $\mathcal{C}$. In fact, a map $f: \mathcal{C} \rightarrow \mathcal{C}^{\prime}$ defines a functor $f^{*}: \operatorname{Cor}_{\mathcal{C}^{\prime}, \mathcal{D}}^{c o c} \rightarrow \operatorname{Cor}_{\mathcal{C}, \mathcal{D}}^{c o c}$ carrying $X \in \operatorname{Cor}_{\mathcal{C}^{\prime}, \mathcal{D}}^{c o c}$ to the composition $X \circ \mathcal{E}_{f}$. Similarly, $g: \mathcal{D} \rightarrow \mathcal{D}^{\prime}$ defines a functor $g_{*}: \operatorname{Cor}_{\mathcal{C}, \mathcal{D}}^{c o c} \rightarrow \operatorname{Cor}_{\mathcal{C}, \mathcal{D}^{\prime}}^{c o c}$ carrying $X \in \operatorname{Cor}_{\mathcal{C}, c D}^{c o c}$ to the composition $\mathcal{E}_{g} \circ X$. Actually, the map $\partial: \operatorname{Cor}^{c o c} \rightarrow$ Cat $\times$ Cat is a bifibration in the sense of [H.EY, 2.2.6]; the proof is given in Subsection 3.1.3 below, after a reminder of the relevant notions.

\subsubsection{Bifibrations. Lax bifibrations}

Let $p: X \rightarrow B \times C$ be a functor. We denote by $p_{B}$ and $p_{C}$ its compositions with the projections to $B$ and $C$, and $X_{b, c}$ (resp., $X_{b \bullet}$ or $X_{\bullet c}$ ) the fiber of $p$ at $(b, c)$ (resp, the fiber of $p_{b}$ at $b$ or the fiber of $p_{C}$ at $\left.c\right)$.

Definition 3.1. The functor $p: X \rightarrow B \times C$ is called a lax bifibration if

(1) $p_{B}$ is a cartesian fibration and $p_{C}$ is a cocartesian fibration.

(2) $p$ is a morphism of cartesian fibrations over $B$, as well as of cocartesian fibrations over $C$.

If $p: X \rightarrow B \times C$ is a lax bifibration, the restriction $p_{C}: X_{b \bullet} \rightarrow C$ is a cocartesian fibration and $p_{B}: X_{\bullet c} \rightarrow B$ is a cartesian fibration.

Lemma 3.2. The following conditions on $p$ are equivalent:

(1) $p_{C}$ is a cocartesian fibration, $p$ is a map of cocartesian fibrations over $C$, and for any $c \in C$ the restriction of $p_{B}$ to $X_{\bullet c}$ is a cartesian fibration. 
(2) $p_{B}$ is a cartesian fibration, $p$ is a map of cartesian fibrations over $B$, and for any $b \in B$ the restriction of $p_{C}$ to $X_{b}$ is a cocartesian fibration.

(3) $p$ is a lax bifibration.

Proof. Let us show that (1) implies (2).

In the formulas below we use the following notation. Given $p: X \rightarrow B \times C, x, y \in$ $X$ and $f: p(x) \rightarrow p(y)$, we denote $\operatorname{Map}^{f}(x, y)$ the fiber of the map $\operatorname{Map}_{X}(x, y) \rightarrow$ $\operatorname{Map}_{B \times C}(p(x), p(y))$ at $f$; we denote $x \rightarrow f_{!}(x)$ the cocartesian lifting of $f$, and $f^{!}(y) \rightarrow y$ the cartesian lifting of $f$ (if it exists).

For arrows $\beta: b \rightarrow b^{\prime}$ in $B$ and $\gamma: c \rightarrow c^{\prime}$ in $C$, and for $x \in X_{b, c}$ and $y \in X_{b^{\prime}, c^{\prime}}$ one has $\operatorname{Map}^{(\beta, \gamma)}(x, y)=\operatorname{Map}^{\left(\beta, c^{\prime}\right)}(\gamma ! x, y)=\operatorname{Map}^{\left(b, c^{\prime}\right)}\left(\gamma ! x, \beta^{!} y\right)$. This formula immediately implies that the map $\beta^{!}(y) \rightarrow y$ is locally $p_{B}$-cartesian. Since composition of such arrows has the same form, $p_{B}$ is a cartesian fibration. The rest of the properties, as well as the opposite implication, are clear.

A lax bifibration can be described as a functor $B^{\mathrm{op}} \rightarrow$ Cat $/ C$ carrying each object of $B^{\text {op }}$ to a cocartesian fibration over $C$, or, equivalently, as a functor $C \rightarrow \mathrm{Cat}_{/ B}$ carrying each object of $C$ to a cartesian fibration over $B$.

Lemma 3.3. Let $p: X \rightarrow B \times C$ be a lax bifibration. Then

- $p^{[1]}: X^{[1]} \rightarrow B^{[1]} \times C^{[1]}$ is also a lax bifibration.

- The fiber $X_{\beta, \gamma}^{[1]}$ of $p^{[1]}$, with $\beta: b \rightarrow b^{\prime}$ and $\gamma: c \rightarrow c^{\prime}$ arrows in $B$ and $C$, is naturally equivalent to the fiber product

$$
X_{b, c} \times_{X_{b, c^{\prime}}} X_{b, c^{\prime}}^{[1]} \times_{X_{b, c^{\prime}}} X_{b^{\prime}, c^{\prime}} .
$$

Proof. The functor Fun([1], _) is well known to preserve cartesian and cocartesian fibrations, see, for instance, $[$ H.lec, 9.6.3]. This implies the first claim of the lemma. The fiber $X_{\beta, \gamma}^{[1]}$ is the category of sections $\operatorname{Fun}_{B \times C}([1], X)$, with $[1] \rightarrow B \times C$ given by $(\beta, \gamma)$. Recall that, for a cocartesian fibration $p: E \rightarrow[1]$ classified by a functor $E_{0} \rightarrow E_{1}, E_{i}=p^{-1}(i)$ for $i=0,1$, the category of sections is described by the formula

$$
\operatorname{Fun}_{[1]}([1], E)=E_{0} \times_{E_{1}} E_{1}^{[1]},
$$

see, for instance, [H.lec, 9.8.5]. We get the formula (1) for lax bifibrations by applying the formula (2) (and a similar formula for cartesian fibrations) twice. First of all, since $p_{B}$ is a cartesian fibration, we get

$$
X_{\beta \bullet}^{[1]}=X_{b \bullet}^{[1]} \times_{X_{b} \bullet} X_{b^{\prime} \bullet}
$$

The category $X_{\beta, \gamma}^{[1]}$ that we wish to describe is the fiber at $\gamma$ of (3), so that

$$
X_{\beta, \gamma}^{[1]}=X_{b, \gamma}^{[1]} \times_{X_{b, c^{\prime}}} X_{b^{\prime}, c^{\prime}}
$$

Finally, since the restriction of $p_{C}$ to $X_{b}$ is a cocartesian fibration, one gets

$$
X_{b, \gamma}^{[1]}=X_{b, c} \times_{X_{b, c^{\prime}}} X_{b, c^{\prime}}^{[1]} .
$$

This proves the lemma.

Definition 3.4. A map $p: X \rightarrow B \times C$ is called a bifibration if it is a lax bifibration and the following equivalent extra conditions are fulfilled: 


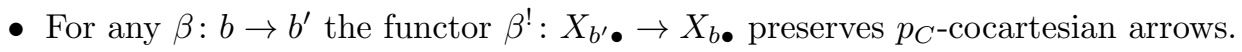

- For any $\gamma: c \rightarrow c^{\prime}$ the functor $\gamma_{!}: X_{\bullet c} \rightarrow X_{\bullet c^{\prime}}$ preserves $p_{B}$-cartesian arrows.

Thus, a bifibration $p: X \rightarrow B \times C$ is classified by a functor $B^{\text {op }} \times C \rightarrow$ Cat.

\subsection{3.}

Let us calculate $\operatorname{Map}\left(\mathcal{E}_{f}, \mathcal{E}_{f^{\prime}}\right)$ for $f: \mathcal{C} \rightarrow \mathcal{D}$ and $f^{\prime}: \mathcal{C}^{\prime} \rightarrow \mathcal{D}^{\prime}$. We have $\mathcal{E}_{f}=(\mathcal{C} \times$ $[1]) \sqcup^{\mathcal{E}} \mathcal{D}$, so the result can be calculated as a fiber product. We have

$$
\operatorname{Map}\left(\mathcal{C} \times[1], \mathcal{E}_{f^{\prime}}\right)=\operatorname{Map}\left(\mathcal{C}, \operatorname{Fun}_{[1]}\left([1], \mathcal{E}_{f^{\prime}}\right)\right)=\operatorname{Map}\left(\mathcal{C}, \mathfrak{C}^{\prime} \times_{\mathcal{D}^{\prime}} \mathcal{D}^{\prime[1]}\right),
$$

see the formula (2). Therefore,

$$
\operatorname{Map}\left(\mathcal{E}_{f}, \mathcal{E}_{f^{\prime}}\right)=\operatorname{Map}\left(\mathcal{C}, \mathcal{C}^{\prime}\right) \times_{\operatorname{Map}\left(\mathcal{C}, \mathcal{D}^{\prime}\right)} \operatorname{Map}\left(\mathcal{C}, \mathcal{D}^{\prime[1]}\right) \times_{\operatorname{Map}\left(\mathcal{C}, \mathcal{D}^{\prime}\right)} \operatorname{Map}\left(\mathcal{D}, \mathcal{D}^{\prime}\right) .
$$

The formula (4) shows that, for any $f: \mathcal{C} \rightarrow \mathcal{C}^{\prime}, g: \mathcal{D} \rightarrow \mathcal{D}^{\prime}$ and $X \in \operatorname{Cor}_{\mathcal{C}^{\prime}, \mathcal{D}}^{c o c}$, the morphism $X \circ \mathcal{E}_{f} \rightarrow X$ is a cartesian lifting of $f$, whereas $X \rightarrow \mathcal{E}_{g} \circ X$ is a cocartesian lifting of $g$. This proves the following.

Lemma 3.5. The map $\partial: \operatorname{Cor}^{c o c} \rightarrow$ Cat $\times$ Cat is a bifibration.

\subsection{Kan extensions}

Let $f: \mathcal{C} \rightarrow \mathcal{D}$ be a functor. A right extension of $f$ with respect to $q: \mathcal{C} \rightarrow \mathcal{C}^{\prime}$ is a functor $f^{\prime}: \mathcal{C}^{\prime} \rightarrow \mathcal{D}$, endowed with a morphism of functors $\theta: f^{\prime} \circ q \rightarrow f$. A right Kan extension is, by definition, a terminal object in the category of right extensions, see [L.T, 4.3.3] for the version for quasicategories.

The category $\mathrm{RE}_{q}(f)$ is defined, therefore, as the fiber product

$$
\operatorname{RE}_{q}(f)=\operatorname{Fun}\left(\mathcal{C}^{\prime}, \mathcal{D}\right) \times_{\operatorname{Fun}(\mathcal{C}, \mathcal{D})} \operatorname{Fun}\left(\mathcal{C}, \mathcal{D}^{[1]}\right) \times_{\operatorname{Fun}(\mathcal{C}, \mathcal{D})}\{f\},
$$

where the map $\operatorname{Fun}\left(\mathfrak{C}^{\prime}, \mathcal{D}\right) \rightarrow \operatorname{Fun}(\mathcal{C}, \mathcal{D})$ is given by composition with $q$.

Definition 3.6. A right Kan extension of $f: \mathcal{C} \rightarrow \mathcal{D}$ with respect to $q: \mathcal{C} \rightarrow \mathcal{C}^{\prime}$ is a terminal object in $\mathrm{RE}_{q}(f)$.

\subsection{1.}

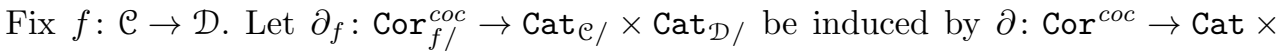
Cat. Denote $\left(\operatorname{Cor}_{/ f}^{c o c}\right)_{q}$ the fiber of $\partial_{f}$ at $\left(q, \operatorname{id}_{\mathcal{D}}\right)$.

In Section 3.3 below we prove the following.

Proposition 3.7. One has a natural equivalence $\mathrm{RE}_{q}(f)^{\mathrm{op}}=\left(\operatorname{Cor}_{f /}^{c o c}\right)_{q}$.

This allows one to redefine a right Kan extension of $f: \mathcal{C} \rightarrow \mathcal{D}$ with respect to $q: \mathcal{C} \rightarrow \mathcal{C}^{\prime}$ as the initial object in $\left(\operatorname{Cor}_{f /}^{c o c}\right)_{q}$.

In other words, a right extension of $f$ along $q$ is an arrow $\theta: \mathcal{E}_{f} \rightarrow \mathcal{E}_{f^{\prime}}$ in $\operatorname{Cor}^{c o c}$, with $\partial_{0}(\theta)=q, \partial_{1}(\theta)=\mathrm{id}_{\mathcal{D}}$.

A right Kan extension is an initial object in the category of such $\theta$ 's. 


\subsection{Construction of equivalence}

We will now identify the bifibration $\mathrm{Cor}^{\mathrm{coc}}$ given by a functor $\mathrm{Cat}^{\mathrm{op}} \times \mathrm{Cat} \rightarrow \mathrm{Cat}$ carrying $(\mathcal{C}, \mathcal{D})$ to $\operatorname{Cor}_{\mathcal{C}, \mathcal{D}}^{c o c}$, with the opposite to the internal Hom in Cat.

Lemma 3.8. There is an equivalence

$$
\operatorname{Fun}(\mathcal{C}, \mathcal{D})^{\mathrm{op}}=\operatorname{Cor}_{\mathcal{C}, \mathcal{D}}^{c o c}
$$

functorial in $\mathcal{C}, \mathcal{D}$.

To deduce Proposition 3.2.1, we apply Lemma 3.3 to Cor $^{c o c}$ and the arrow [1] $\rightarrow$ Cat $\times$ Cat given by the pair $\left(q, \mathrm{id}_{\mathcal{D}}\right)$.

Proof of Lemma 3.8. Recall an important way of presentation of categories going back to Rezk's CSS model structure for $\infty$-categories.

The embedding $\Delta \rightarrow$ Cat defines a functor $\mathcal{N}$ : Cat $\rightarrow P(\Delta)=\operatorname{Fun}\left(\Delta^{\mathrm{op}}, \mathcal{S}\right)$ assigning to a category $\mathcal{C}$ its "Rezk nerve", a simplicial space carrying $[n]$ to $\operatorname{Map}([n], \mathcal{C})$. The functor $\mathcal{N}$ is fully faithful; its image consists of simplicial spaces that are Segal and complete. Moreover, this embedding has a left adjoint $L: \operatorname{Fun}\left(\Delta^{\mathrm{op}}, \mathcal{S}\right) \rightarrow$ Cat which is a localization. ${ }^{4}$

Let us describe the simplicial space $\mathcal{N}(\operatorname{Fun}(\mathcal{C}, \mathcal{D}))$. One has

$$
\operatorname{Map}([n], \operatorname{Fun}(\mathcal{C}, \mathcal{D}))=\operatorname{Map}_{[n]}(\mathcal{C} \times[n], \mathcal{D} \times[n]) .
$$

Lemma $3.2,(1)$, shows that this is the space of lax bifibrations $p: X \rightarrow[n] \times[1]$ satisfying the conditions

$$
X_{\bullet 0}=[n] \times \mathcal{C} \text { and } X_{\bullet 1}=[n] \times \mathcal{D} .
$$

Let us now describe $\mathcal{N}\left(\operatorname{Cor}_{\mathcal{C}, \mathcal{D}}^{c o c}\right)$. We use the cartesian version of the Grothendieck construction and Lemma 3.2, $(2)$, to describe $\operatorname{Map}\left([n], \operatorname{Cor}_{\mathcal{C}, \mathcal{D}}^{c o c}\right)$ as the space of lax bifibrations $p: X \rightarrow[n]^{\text {op }} \times[1]$ satisfying the conditions (5). Using the canonical isomorphism $[n]=[n]^{\text {op }}$, we get the required equivalence.

\section{Main results}

The results listed below are mostly direct consequences of the constructions of Section 3.

Lemma 4.1. Let $f: \mathcal{C} \rightarrow \mathcal{D}$ be as above, and let $q_{\mathcal{C}}: \mathcal{C} \rightarrow \mathcal{C}^{\prime}$ and $q_{\mathcal{D}}: \mathcal{D} \rightarrow \mathcal{D}^{\prime}$ be localizations. Let $\mathcal{E}_{f}^{\prime}$ be the localization of $\mathcal{E}_{f}$ described in Section 2.3 and let $\theta: \mathcal{E}_{f} \rightarrow$ $\mathcal{E}_{f^{\prime}}$ be a right extension of $q_{\mathcal{D}} \circ f$ along $q_{\mathcal{C}}$. Then $\theta$ uniquely factors through $\mathcal{E}_{f}^{\prime}$.

We denote the unique map $\mathcal{E}_{f}^{\prime} \rightarrow \mathcal{E}_{f^{\prime}}$ as $\theta^{\prime}$.

Corollary 4.2. Let $f: \mathcal{C} \rightarrow \mathcal{D}$ admit a left derived functor $\mathbf{L} f$. Then the canonical equivalence $\mathcal{E}_{f}^{\prime} \rightarrow \mathcal{E}_{\mathbf{L} f}$ presents $\mathcal{E}_{\mathbf{L} f}$ as a right Kan extension of $q_{\mathcal{D}} \circ f$ along $q_{\mathfrak{C}}$.

That is, our left derived functors are right Kan extensions, and right derived functors are left Kan extension. ${ }^{5}$ Note that our derived functors may not exist, even when Kan extensions exist. Note also, that

\footnotetext{
${ }^{4}$ A localization having fully faithful right adjoint is called a Bousfield localization.

${ }^{5}$ It is very easy to see that they are actually absolute Kan extensions.
} 
Proposition 4.3. Let $f: \mathcal{C} \rightarrow \mathcal{D}$ be a functor, let $W_{\mathcal{C}}$ and $W_{\mathcal{D}}$ be subcategories of $\mathcal{C}$ and $\mathcal{D}$ respectively, and let $f\left(W_{\mathcal{C}}\right) \subset W_{\mathcal{D}}$. Then the composition $q \circ f: \mathcal{C} \rightarrow \mathcal{D}^{\prime}$ factors uniquely through a functor $f^{\prime}: \mathcal{C}^{\prime} \rightarrow \mathcal{D}^{\prime}$ which is both a left and right derived functor of $f$.

Proof. We have to verify that the canonical map $\theta^{\prime}: \mathcal{E}_{f}^{\prime} \rightarrow \mathcal{E}_{f^{\prime}}$ is an equivalence. By universality of localization,

$$
\mathcal{E}_{f}^{\prime}=\mathcal{C}^{\prime} \sqcup^{\mathcal{e}}(\mathcal{C} \times[1]) \sqcup^{\mathcal{e}} \mathcal{D}^{\prime}
$$

with the map $\mathcal{C} \rightarrow \mathcal{D}^{\prime}$ given by a composition $q \circ f$. We will show that the natural map from the expression above to $\mathcal{E}_{f^{\prime}}=\left(\mathfrak{C}^{\prime} \times[1]\right) \sqcup^{\mathcal{C}^{\prime}} \mathcal{D}^{\prime}$ is an equivalence. It is enough, for any $X$, to prove that the induced map

$$
\operatorname{Fun}\left(\varepsilon_{f^{\prime}}, X\right) \rightarrow \operatorname{Fun}\left(\varepsilon_{f}^{\prime}, X\right)
$$

is an equivalence. It is easy to see that, by universality of localization, both identify with the full subcategory of $\operatorname{Fun}\left(\varepsilon_{f}, X\right)$ spanned by the functors carrying $W_{\mathcal{e}}$ and $W_{\mathcal{D}}$ to equivalences in $X$.

\subsection{Existence}

We will now prove that derived functors exist reasonably often.

Keeping the previous notation, let now $i: \mathcal{C}_{0} \rightarrow \mathcal{C}$ be a functor. We define $W_{0}=$ $i^{-1}\left(W_{\mathcal{C}}\right)$ and we assume that the composition $f \circ i: \mathcal{C}_{0} \rightarrow \mathcal{D}$ preserves weak equivalences. The commutative diagram

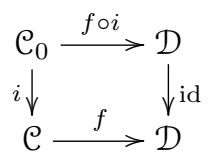

gives rise to a map $\alpha: \mathcal{E}_{f \circ i} \rightarrow \mathcal{E}_{f}$ in Cor, and, after localization, to a map $\alpha^{\prime}: \mathcal{E}_{f \circ i}^{\prime} \rightarrow \mathcal{E}_{f}^{\prime}$. Proposition 4.4. Let $f: \mathcal{C} \rightarrow \mathcal{D}, W_{\mathcal{C}}$ and $W_{\mathcal{D}}$ be as above. Assume there exists a functor $i: \mathcal{C}_{0} \rightarrow \mathcal{C}$ satisfying the following properties:

- The composition $f \circ i: \mathcal{C}_{0} \rightarrow \mathcal{D}$ preserves weak equivalences.

- The map $i$ induces an equivalence $i^{\prime}: \mathcal{C}_{0}^{\prime} \rightarrow \mathcal{C}^{\prime}$.

- A right Kan extension of $q_{\mathcal{D}} \circ f$ along $q_{\mathcal{C}}$ exists. It is given by a map $\theta: \mathcal{E}_{f} \rightarrow$ $\mathcal{E}_{f^{\prime}}$, where $f^{\prime}: \mathcal{C}^{\prime} \rightarrow \mathcal{D}^{\prime}$, such that the composition $\theta^{\prime} \circ \alpha^{\prime}: \mathcal{E}_{f \circ i}^{\prime} \rightarrow \mathcal{E}_{f}^{\prime} \rightarrow \mathcal{E}_{f^{\prime}}$ is an equivalence.

Then the left derived functor $\mathbf{L} f$ exists (and can be calculated as $\left.(f \circ i)^{\prime} \circ i^{\prime^{-1}}\right)$.

Proof. We have to verify that the canonical map $\theta^{\prime}: \mathcal{E}_{f}^{\prime} \rightarrow \mathcal{E}_{f^{\prime}}$ obtained by the localization of the right Kan extension $\theta: \mathcal{E}_{f} \rightarrow \mathcal{E}_{f^{\prime}}$, is an equivalence. Since $\theta^{\prime} \circ \alpha^{\prime}$ is an equivalence, its inverse, composed with $\alpha^{\prime}: \mathcal{E}_{f \circ i}^{\prime} \rightarrow \mathcal{E}_{f}^{\prime}$, yields a map $\mathcal{E}_{f^{\prime}} \rightarrow \mathcal{E}_{f}^{\prime}$ in the opposite direction.

Both compositions are equivalent to the identity as $\mathcal{E}_{f^{\prime}}$ is universal in $\left(\operatorname{Cor}_{f /}^{c o c}\right)_{q}$ and $\mathcal{E}_{f}^{\prime}$ is universal in $\left(\operatorname{Cor}_{f /}\right)_{q}:=\operatorname{Cor}_{f /} \times_{\operatorname{Cat}_{\mathcal{e} /} \times \operatorname{Cat}_{\mathcal{D} /}}\left\{\left(q, \mathrm{id}_{\mathcal{D}}\right)\right\}$.

Let now $f: \mathcal{C} \rightarrow \mathcal{D}$ be a functor between model categories. Applying Proposition 4.4 to $\mathcal{C}_{0}$, the subcategory of fibrant (resp., cofibrant) objects in $\mathcal{C}$, we get the following. 
Corollary 4.5. Left (resp., right) Quillen functors admit a left (resp., right) derived functor in the sense of Definition 2.1 (resp., Definition 2.2).

\subsection{Functoriality}

\subsubsection{Flat fibrations}

Recall that a functor $p: \mathcal{E} \rightarrow[2]$ is called a flat fibration if the natural map

$$
\mathcal{E}_{\{0,1\}} \sqcup^{\mathcal{E}_{1}} \mathcal{E}_{\{1,2\}} \rightarrow \mathcal{E}
$$

where $\mathcal{E}_{i}$, resp., $\mathcal{E}_{\{i, j\}}$, are defined by base change of $\mathcal{E}$ with respect to $\{i\} \rightarrow[2]$, resp., $\{i, j\} \rightarrow[2]$, is an equivalence. More generally, a map $p: \mathcal{E} \rightarrow B$ is a flat fibration if any base change of $p$ with respect to [2] $\rightarrow B$ is flat in the above sense. An important property of flat fibrations, see [L.HA, B.4.5], says that, if $\mathcal{E} \rightarrow B$ is flat, the base change functor $\mathrm{Cat}_{/ B} \rightarrow \mathrm{Cat}_{/ \mathcal{E}}$ preserves colimits.

\subsection{2.}

A derived functor of a composition is not, in general, the composition of derived functors. It is interesting to see what is going on here, in terms of correspondences. A composable pair of functors $f: \mathcal{E}_{0} \rightarrow \mathcal{E}_{1}, g: \mathcal{E}_{1} \rightarrow \mathcal{E}_{2}$ is given by a cocartesian fibration $p: \mathcal{E} \rightarrow[2]$. Given a subcategory $\mathcal{W} \subset \mathcal{E}$ over [2] ${ }^{e q}=\{0\} \cup\{1\} \cup\{2\}$, the localization $\mathcal{E}^{\prime}$ is flat over [2], as the universal property of localization gives the presentation

$$
\mathcal{E}^{\prime}=\mathcal{E}_{0}^{\prime} \sqcup^{\mathcal{E}_{0}} \mathcal{E}_{\{0,1\}} \sqcup^{\mathcal{E}_{1}} \mathcal{E}_{1}^{\prime} \sqcup^{\mathcal{E}_{1}} \mathcal{E}_{\{1,2\}} \sqcup^{\mathcal{E}_{2}} \mathcal{E}_{2}^{\prime},
$$

as well as presentations

$$
\mathcal{E}_{0,1}^{\prime}=\mathcal{E}_{0}^{\prime} \sqcup^{\varepsilon_{0}} \mathcal{E}_{\{0,1\}} \sqcup^{\mathcal{E}_{1}} \mathcal{E}_{1}^{\prime} \text { and } \varepsilon_{1,2}^{\prime}=\mathcal{E}_{1}^{\prime} \sqcup^{\mathcal{E}_{1}} \mathcal{E}_{\{1,2\}} \sqcup^{\varepsilon_{2}} \mathcal{E}_{2}^{\prime} .
$$

If $\mathbf{L} f$ and $\mathbf{L} g$ exist, $\mathcal{E}^{\prime}$ is therefore a cocartesian fibration, that is, it is classified by the pair of functors $\mathbf{L} f, \mathbf{L} g$.

The base change of the localization map $\mathcal{E} \rightarrow \mathcal{E}^{\prime}$ with respect to $\delta^{1}:[1] \rightarrow[2]$ yields a map

$$
\mathcal{E}_{g \circ f} \rightarrow \mathcal{E}_{\mathbf{L} g \circ \mathbf{L} f}
$$

which induces a map

$$
\mathcal{E}_{g \circ f}^{\prime} \rightarrow \mathcal{E}_{\mathbf{L} g \circ \mathbf{L} f}
$$

This map is not necessarily an equivalence. ${ }^{6}$

If the canonical map (6) is an equivalence, the left derived functor of $g \circ f$ is defined and $\mathbf{L} g \circ \mathbf{L} f=\mathbf{L}(g \circ f)$.

\subsubsection{Deriving a family of functors}

Given a functor $F: B \rightarrow$ Cat, we can convert it into a cocartesian fibration $p: \mathcal{E} \rightarrow B$. Given a subcategory $W$ in $p^{-1}\left(B^{e q}\right)$, we can define $p^{\prime}: \mathcal{E}^{\prime} \rightarrow B$ as the localization of $\mathcal{E}$ with respect to $W$.

Definition 4.6. We say that the functor $F$ is left derivable with respect to $W$ if

- $p^{\prime}: \mathcal{E}^{\prime} \rightarrow B$ is a cocartesian fibration.

\footnotetext{
${ }^{6}$ Thus, the localization does not always commute with the base change.
} 
- For each $a:[1] \rightarrow B$ the base change of $\mathcal{E} \rightarrow \mathcal{E}^{\prime}$ with respect to a remains a localization.

Thus, a derivable functor $F: B \rightarrow$ Cat gives rise to a new functor $F^{\prime}: B \rightarrow$ Cat defined by the formulas

$$
F^{\prime}(b)=F(b)^{\prime}, F^{\prime}(\alpha)=\mathbf{L} F(\alpha) .
$$

It is obvious that, when $F: B \rightarrow$ Cat is left derivable, one has an equivalence

$$
\mathbf{L} F(\gamma) \stackrel{\sim}{\rightarrow} \mathbf{L} F(\beta) \circ \mathbf{L} F(\alpha)
$$

for any commutative triangle with edges $\alpha, \beta$ and $\gamma=\beta \circ \alpha$ in $B$. The following result shows that the converse is also true.

Proposition 4.7. Let $p: \mathcal{E} \rightarrow B$ be a cocartesian fibration classified by a functor $F: B \rightarrow$ Cat. Let $W$ be a subcategory of $p^{-1}\left(B^{e q}\right)$. Assume that

1. For any $a:[1] \rightarrow B$ the composition $F \circ a:[1] \rightarrow$ Cat defines a functor having a left derived functor with respect to $W$.

2. For any $b:[2] \rightarrow B$ with edges $\alpha, \beta$ and $\gamma=\beta \circ \alpha$, the natural map

$$
\mathbf{L} F(\gamma) \stackrel{\sim}{\rightarrow} \mathbf{L} F(\beta) \circ \mathbf{L} F(\alpha)
$$

is an equivalence.

Then $F$ is left derivable with respect to $W$.

Proof. The proof will proceed as follows. First of all, we will verify the claim for $B=[n]$. Then we will deduce it for a general $B$, using a presentation of $B$ as a colimit.

The claim is vacuous for $B=[0]$, [1]. Let us verify it for $B=[n]$. The maps $s:[1] \rightarrow[n]$ and $t:[n-1] \rightarrow[n]$ given by the formulas $s(0)=0, s(1)=1, t(i)=$ $i+1$, are flat. $\mathcal{E}^{\prime}$ is obtained by localizing $\mathcal{E}$ with respect to $W=\sqcup W_{i}$ where $W_{i} \subset$ $\mathcal{E}_{i}=p^{-1}(\{i\}), i \in[n]$. This allows one to present $\mathcal{E}^{\prime}$ as a colimit of the diagram

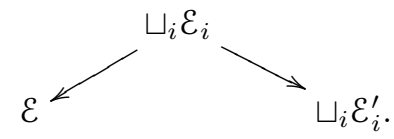

The base change with respect to flat $s$ and $t$ preserves colimits. We deduce that, by induction, $p^{\prime}: \mathcal{E}^{\prime} \rightarrow[n]$ is a cocartesian fibration.

Now, given $c:[1] \rightarrow[n]$, we have to verify that the base change of $\mathcal{E} \rightarrow \mathcal{E}^{\prime}$ with respect to $c, \mathcal{E}_{c} \rightarrow \mathcal{E}_{c}^{\prime}$, is a localization. By induction, it is sufficient to assume that $c(0)=0, c(1)=n$. Look at the commutative triangle $\gamma=\beta \circ \alpha$ in $[n]$ with $\gamma: 0 \rightarrow$ $n, \alpha: 0 \rightarrow 1$ and $\beta: 1 \rightarrow n$. We denote by $a:[1] \rightarrow[n]$ and $b:[1] \rightarrow[n]$ the maps corresponding to $\alpha$ and $\beta$.

By induction we can assume that $\mathcal{E}_{a} \rightarrow \mathcal{E}_{a}^{\prime}$ and $\mathcal{E}_{b} \rightarrow \mathcal{E}_{b}^{\prime}$ are localizations, which gives, by Condition 2 applied to the commutative triangle $\gamma=\beta \circ \alpha$, that $\mathcal{E}_{c} \rightarrow \mathcal{E}_{c}^{\prime}$ is a localization.

We now treat the case of arbitrary $B$. Recall that the Grothendieck construction provides a canonical identification between the category of cocartesian fibrations $\operatorname{Coc}(B)$ over $B$ and the category of functors $\operatorname{Fun}(B$, Cat). This implies that, 
for $B=\operatorname{colim} B_{i}$, one has an equivalence $\eta: \operatorname{Coc}(B) \rightarrow \lim \operatorname{Coc}\left(B_{i}\right)$. The map $\eta$ is defined by the compatible collection of base change maps $\operatorname{Coc}(B) \rightarrow \operatorname{Coc}\left(B_{i}\right)$ for each $B_{i} \rightarrow B$. Let us describe a quasi-inverse map $\theta: \lim \operatorname{Coc}\left(B_{i}\right) \rightarrow \operatorname{Coc}(B)$. Given a compatible collection of cocartesian fibrations $\mathcal{E}_{i} \rightarrow B_{i}$, it assigns a cocartesian fibration $p: \mathcal{E} \rightarrow B$ endowed with a compatible collection of equivalences $\mathcal{E}_{i} \rightarrow B_{i} \times_{B} \mathcal{E}$. Since $p: \mathcal{E} \rightarrow B$ is flat, the fiber product $\times_{B} \mathcal{E}$ preserves colimits, so the collection of maps $\mathcal{E}_{i} \rightarrow \mathcal{E}$ is a colimit diagram. Thus, $\theta\left(\left\{\mathcal{E}_{i}\right\}\right)=\operatorname{colim} \mathcal{E}_{i}$.

Thus, given a compatible collection of cocartesian fibrations $p_{i}: \mathcal{E}_{i} \rightarrow B_{i}$, one has $\mathcal{E}_{i}=\mathcal{E} \times{ }_{B} B_{i}$ where $B=\operatorname{colim} B_{i}$ and $\mathcal{E}=\operatorname{colim} \mathcal{E}_{i}$.

Let now $\Delta_{/ B}$ denote the full subcategory of Cat $/ B$ spanned by the arrows $[n] \rightarrow B$. Denote by $\pi: \Delta_{/ B} \rightarrow$ Cat the functor carrying $a:[n] \rightarrow B$ to $[n] \in$ Cat. It is standard ${ }^{7}$ that $B=\operatorname{colim}(\pi)$.

Therefore, the functor $\rho: \Delta_{/ B} \rightarrow$ Cat, carrying $a:[n] \rightarrow B$ to $\mathcal{E}_{a}=[n] \times_{B} \mathcal{E}$, has the colimit $\mathcal{E}=\operatorname{colim}(\rho)$. Recall that $\mathcal{E}$ has a marking defined by the subcategory $W \subset p^{-1}\left(B^{e q}\right)$. The arrows in $\Delta_{/ B}$ preserve the markings, so $\rho$ is actually a functor from $\Delta_{/ B}$ to $\mathrm{Cat}^{+}$, the category of marked categories, see Subsection 2.3.1. We denote by $\rho^{\prime}: \Delta / B \rightarrow$ Cat the composition of $\rho$ with the localization $\mathcal{L}:$ Cat $^{+} \rightarrow$ Cat. Since localization commutes with colimits, we have $\operatorname{colim}\left(\rho^{\prime}\right)=\mathcal{E}^{\prime}$. This implies that for any $a:[n] \rightarrow B$ the localization of $\mathcal{E}_{a}=[n] \times_{B} \mathcal{E}$ is $\mathcal{E}_{a}^{\prime}=[n] \times{ }_{B} \mathcal{E}^{\prime}$.

\subsubsection{Deriving a family of adjoint pairs of functors}

A family of adjoint pairs of functors is just a functor $F: B \rightarrow$ Cat such that, for each arrow $\alpha: b \rightarrow b^{\prime}$ in $B$, the functor $F(\alpha)$ has a right adjoint. Equivalently, this means that the corresponding cocartesian fibration $p: \mathcal{E} \rightarrow B$ is also a cartesian fibration.

For $W \subset p^{-1}\left(B^{e q}\right)$ let $p^{\prime}: \mathcal{E}^{\prime} \rightarrow B$ be obtained by localizing $p: \mathcal{E} \rightarrow B$ with respect to $W$.

Proposition 4.7 implies the following.

Corollary 4.8. Let, in the notation of Proposition 4.7, $p: \mathcal{E} \rightarrow B$ be a cartesian and cocartesian fibration. Assume as well that, apart of conditions of Proposition 4.7, for each $a:[1] \rightarrow B$ the base change $\mathcal{E} \times_{B}$ [1] has a cartesian and cocartesian localization. Then $p^{\prime}: \mathcal{E}^{\prime} \rightarrow B$ is also a cartesian cocartesian localization(and therefore defines a family of derived adjoint pairs of functors).

\subsection{Deligne's definition}

Let $f: \mathcal{C} \rightarrow \mathcal{D}$ be a functor between conventional categories, and let $W$ be a multiplicative system in $\mathcal{C}$ satisfying the right calculus of fractions in the sense of GabrielZisman [GZ, Section 2].

According to Deligne's approach $\left[\mathbf{D}\right.$, Definition 1.2.1], ${ }^{8}$ the left derived functor $\mathbf{L} f$ is constructed as follows.

\footnotetext{
${ }^{7}$ Cat is a full subcategory of $P(\Delta)$ and $B=\operatorname{colim}(\pi)$ where the colimit is calculated in $P(\Delta)$. To calculate the colimit in Cat, one has to make a complete Segal replacement, which is not needed since $B$ is already complete and Segal simplicial space.

${ }^{8}$ Deligne formulates this notion for triangulated categories.
} 
For $x \in \mathcal{C}$ denote $L_{x}$ the category of arrows $s: x^{\prime} \rightarrow x$ in $\mathcal{C}$ belonging to $W$. Define a functor

$$
f^{\prime}: \mathcal{C}^{\mathrm{op}} \times \mathcal{D} \rightarrow \text { Set }
$$

by the formula

$$
(x, y) \mapsto \operatorname{colim}\left\{\phi_{x, y}: L_{x}^{\mathrm{op}} \rightarrow \operatorname{Set}\right\},
$$

where $\phi_{x, y}\left(s: x^{\prime} \rightarrow x\right)=\operatorname{Hom}_{\mathcal{D}}\left(f\left(x^{\prime}\right), y\right)$. The functor $f^{\prime}$ defines a correspondence from $\mathcal{C}$ to $\mathcal{D}$ which we denote $\mathcal{E}^{D e l}$.

The left derived functor $\mathbf{L} f$ is defined whenever $\mathcal{E}^{D e l}$ is a cocartesian fibration.

In this case $\mathcal{E}^{D e l}$ defines a functor $\mathcal{C} \rightarrow \mathcal{D}$ carrying $W$ to isomorphisms, and so having a unique factorization through the localization $\mathcal{C}^{\prime}$ of $\mathcal{C}$.

Let $\mathcal{E}_{f}$ be the cocartesian fibration classified by the functor $f$. One has an obvious map $\mathcal{E}_{f} \rightarrow \mathcal{E}^{D e l}$ in Cor. Let $\mathcal{E}_{f}^{\prime}$ be the localization of $\mathcal{E}$ with respect to $W$. One has a map $\delta: \mathcal{E}^{\text {Del }} \rightarrow \mathcal{E}_{f}^{\prime}$ defined by the compatible collection of maps $\operatorname{Hom}_{\mathcal{D}}\left(f\left(x^{\prime}\right), y\right) \rightarrow$ $\operatorname{Hom}_{\mathcal{E}_{f}^{\prime}}(x, y)$ carrying $\alpha: f\left(x^{\prime}\right) \rightarrow y$ to the composition

$$
x \stackrel{s^{-1}}{\longrightarrow} x^{\prime} \stackrel{\alpha}{\longrightarrow} y
$$

in $\mathcal{E}_{f}^{\prime}$. The functor $\delta$ induces a functor $\delta^{\prime}:\left(\mathcal{E}^{D e l}\right)^{\prime} \rightarrow \mathcal{E}_{f}^{\prime}$ from the localization of $\mathcal{E}^{D e l}$ with respect to $W$, to $\mathcal{E}_{f}^{\prime}$. This map is fully faithful, as $\left(\mathcal{E}_{f}, W\right)$ satisfies the right calculus of fractions. Therefore, Deligne's definition of derived functor, in case $W$ satisfies a calculus of fractions, coincides with the one defined in Definition 2.1.

\section{4. $\infty$-localization versus conventional localization}

Our definition of derived functor makes sense in both contexts, as we only use the universal property of localizations. Moreover, if a derived functor exists in the infinity setting, its conventional truncation gives a derived functor in the conventional setting.

Proposition 4.9. Let $f: \mathcal{C} \rightarrow \mathcal{D}$ be a functor between conventional categories. Let $\mathrm{C}^{\prime}$ and $\mathcal{D}^{\prime}$ be their $\infty$-localizations, and $\mathrm{Ho}(\mathcal{C}), \operatorname{Ho}(\mathcal{D})$ their conventional truncations obtained by applying the functor $\pi_{0}$ to all function spaces. Assume $f^{\prime}: \mathfrak{C}^{\prime} \rightarrow \mathcal{D}^{\prime}$ is a left derived functor of $f$ in the sense of Definition 2.1. Then the induced conventional functor $\operatorname{Ho}\left(f^{\prime}\right): \operatorname{Ho}\left(\mathrm{C}^{\prime}\right) \rightarrow \operatorname{Ho}\left(\mathcal{D}^{\prime}\right)$ is a left derived functor in the conventional setting.

The claim immediately follows from the following observation.

Lemma 4.10. Let $f: \mathcal{C} \rightarrow \mathcal{D}$ be a functor, and let $p: \mathcal{E}_{f} \rightarrow[1]$ be the corresponding cocartesian fibration. Then the induced map $\operatorname{Ho}(p): \operatorname{Ho}(\mathcal{E}) \rightarrow[1]$ is a cocartesian fibration classified by the functor $\mathrm{Ho}(f): \operatorname{Ho}(\mathcal{C}) \rightarrow \operatorname{Ho}(\mathcal{D})$.

Proof. The commutative square

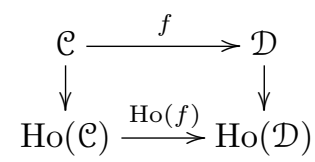

induces a map $\mathcal{E}_{f} \rightarrow \mathcal{E}_{\mathrm{Ho}(f)}$ in $\operatorname{Coc}([1])$. The map $\mathrm{Ho}\left(\mathcal{E}_{f}\right) \rightarrow[1]$ is a cocartesian fibration as the image in $\operatorname{Ho}\left(\mathcal{E}_{f}\right)$ of any cocartesian arrow in $\mathcal{E}_{f}$ is cocartesian. Therefore, 
a map of cocartesian fibrations $\operatorname{Ho}\left(\mathcal{E}_{f}\right) \rightarrow \mathcal{E}_{\mathrm{Ho}(f)}$ is induced. It is an equivalence as it induces an equivalence of the fibers.

\section{References}

[C] D.-C. Cisinski, Higher categories and homotopical algebra, http://www . mathematik.uni-regensburg.de/cisinski/CatLR.pdf, the manuscript to appear in Cambridge Studies in Advanced Mathematics, Cambridge University Press.

[D] P. Deligne, Cohomologie à support propre, SGA 4, exposé XVII, Lecture Notes in Mathematics, vol. 305 (1973), Springer.

[GZ] P. Gabriel, M. Zisman, Calculus of fractions and homotopy theory, Ergebnisse der Mathematik und ihrer Grenzgebiete, Band 35 (1967), Springer.

[RD] R. Hartshorne, Residues and duality, Lecture notes of a seminar on the work of A. Grothendieck, Harvard 1963/64, Lecture Notes in Mathematics, vol. 20 (1966), Springer.

[H.EY] V. Hinich, Yoneda lemma for enriched $\infty$-categories, Adv. Math. 367 (2020).

[H.L] V. Hinich, Dwyer-Kan localization revisited, Homology Homotopy Appl. 18 (2016), no. 1, 27-48.

[H.lec] V. Hinich, Lectures on infinity categories, arXiv:1709.06271, $125 \mathrm{pp}$.

[HS] A. Hirschowitz, C. Simpson, Descente pour les $n$-champs, arXiv:math/ 9807049v3.

[K] B. Keller, Derived categories and their uses, Handbook of algebra, Vol. 1, 671-701, Elsevier/North-Holland, Amsterdam, 1996.

[L.HA] J. Lurie, Higher algebra, preprint September 18, 2017, available at http:// www . math.harvard.edu/ lurie/papers/HA.pdf.

[L.T] J. Lurie, Higher topos theory, Annals of Mathematics Studies, vol. 170 (2009), Princeton University Press, Princeton, NJ, xviii+925 pp.

[Mal] G. Maltsiniotis, Le théorème de Quillen d'adjonction de foncteurs dérivés, revisité, C.R. Acad. Sci. Paris, Ser. I 344 (2007), 549-552.

[Me] N. Meadows, Descent theory and mapping spaces, arXiv:1809.00592v2.

[Q.HA] D. Quillen, Homotopical algebra, Lecture Notes in Mathematics, vol. 43 (1967), Springer.

Vladimir Hinich hinich@math.haifa.ac.il

Department of Mathematics, University of Haifa, Mount Carmel, Haifa 3498838, Israel 